\title{
RNA interference: The molecular immune system
}

\author{
Omar Bagasra $^{1} \&$ Kiley R. Prilliman ${ }^{2}$ \\ ${ }^{1}$ South Carolina Center for Biotechnology, Claflin University, Orangeburg, SC 29115, USA ${ }^{2}$ Baylor Institute \\ for Immunology Research, Dallas, TX 75204, USA
}

Received 5 December 2003 and in revised form 10 June 2004

\begin{abstract}
Summary
Introduction of double-stranded RNA (dsRNA) into cells expressing a homologous gene triggers RNA interference (RNAi), or RNA-based gene silencing (RBGS). The dsRNA degrades corresponding host mRNA into small interfering RNAs (siRNAs) by a protein complex containing Dicer. siRNAs in turn are incorporated into the RNA-induced silencing complex (RISC) that includes helicase, RecA, and exo- and endo-nucleases as well as other proteins. Following its assembly, the RISC guides the RNA degradation machinery to the target RNAs and cleaves the cognate target RNA in a sequence-specific, siRNA-dependent manner. RNAi has now been documented in a wide variety of organisms, including plants, fungi, flies, worms, and more recently, higher mammals. In eukaryotes, dsRNA directed against a range of viruses (i.e., HIV-1, RSV, HPV, poliovirus and others) and endogenous genes can induce sequence-specific inhibition of gene expression. In invertebrates, RNAi can be efficiently triggered by either long dsRNAs or 21- to 23-nt-long siRNAs. However, in jawed vertebrates, dsRNA longer than $30 \mathrm{bp}$ can induce interferon and thus trigger undesirable side effects instead of initiating RNAi. siRNAs have been shown to act as potent inducers of RNAi in cultured mammalian cells. Many investigators have suggested that siRNAs may have evolved as a normal defense against endogenous and exogenous transposons and retroelements. Through a combination of genetic and biochemical approaches, some of the mechanisms underlying RNAi have been described. Recent data in C. elegans shows that two homologs of siRNAs, microRNAs (miRNAs) and tiny noncoding RNAs (tncRNAs) are endogenously expressed. However, many aspects of RNAi-induced gene silencing, including its origins and the selective pressures which maintain it, remain undefined. Its evolutionary history may pass through the more primitive immune functions of prokaryotes involving restriction enzymes that degrade plasmid DNA molecules that enter bacterial cells. RNAi has evolved further among eukaryotes, in which its wide distribution suggests early origins. RNAi seems to be involved in a variety of regulatory and immune functions that may differ among various kingdoms and phyla. We present here proposed mechanisms by which RBGS protects the host against endogenous and exogenous transposons and retroelements. The potential for therapeutic application of RBGS technology in treating viral infections such as HIV is also discussed.
\end{abstract}

\section{Introduction}

Life on the green planet most likely found its roots in RNA, which essentially plays a passive role as a storage database and an active one as a catalyst. As evolution progressed and continually larger amounts of data required management, these roles were assumed by the comparatively more stable DNA, and additional proteins, complex carbohydrates, and lipid molecules took over the tasks of enzymes and structural building blocks. Meanwhile, RNA appeared to take on an intermediate role as translator of the massive amounts of information comprising the DNA database. However, we have recently begun to realize that, throughout evolution, the role of RNA in a discreet yet significant innate anti-viral defense system has been conserved; as such, RNA potentially functions as the major vanguard in preserving the integrity of the host organism's genomic structure via RNA interference (RNAi), or RNA-based gene silencing (RBGS) (reviewed in Fire 1999, Hamilton \& Baulcombe 1999, Sharp 1999, Tuschl 1999, Bass 2000, Grishok \& Mello 2002, Hannon 2002, Hutvager \& Zamore 2002, Lindenbach \& Rice 2002).

The concept of "molecular immunity" results from a fusion of concepts from diverse fields including plant biology, molecular genetics, immunology, and biochemistry; they have contributed to describing a mechanism by which eukaryotes are able to regulate gene expression, fight double-stranded RNA (dsRNA) viral infections, and protect their genomes from genetic parasites such as transposons (the DNA mobile elements that move from one location to another), retrotransposons (the DNA mobile element that use RNA intermediate), and retroviruses (Fire 1999, Hamilton \& Baulcombe 1999, Ketting et al. 1999, Sharp 1999, 
Tabara et al. 1999, Tuschl 1999, Bass 2000, Zamore et al. 2000, Grishok \& Mello 2002, Hannon 2002, Hutvagner \& Zamore 2002, Lindenbach \& Rice 2002, Salo et al. 2002, Urwin et al. 2002, Seitz et al. 2003). In the last several years, numerous examples of RNAbased intracellular molecular defense mechanisms have been described. All of these are epigenetic and are based upon recognition of nucleic acid sequence homology at the mRNA level, the degradation of a homologous RNA by dsRNA, or triplex RNA (Bagasra \& Amjad 1997, Bagasra \& Amjad, 2000). RBGS has been shown to inhibit production of retroviruses (the human immunodeficiency virus, HIV-1 (Bagasra \& Amjad 2000, Jacque et al. 2002), Rous sarcoma virus (reviewed by $\mathrm{Hu}$ et al. 2002, Joost et al. 2003), RNA viruses (respiratory syncytial virus and poliovirus: Joost et al. 2003), and a DNA virus (human papillomavirus (reviewed in Bagasra 1999, Gitlin \& Andino 2003, Joost et al. 2003).

The phenomenon of RNAi was originally detailed in 1998 by Fire et al. (1998) of the Carnegie Institution of Washington (at Johns Hopkins University), and further explored by several leading groups including Sharp and Zamora and their team at MIT (Sharp 1999, Zamore et al. 2000), and Baulcombe and his group at the Sainsbury Laboratory in UK (Hamilton \& Baulcombe 1999). To date, the RNAi effect has been documented in a variety of organisms including plants, fungi, flies, worms, and also complex mammals (Fire 1999, Hamilton \& Baulcombe 1999, Ketting et al. 1999, Sharp 1999, Tabara et al. 1999, Tuschl 1999, Bass 2000, Zamore et al. 2000, Grishok \& Mello 2002, Hannon 2002, Hutvagner \& Zamore 2002, Lindenbach \& Rice 2002, Salo et al. 2002, Urwin et al. 2002). From an immunological perspective, this RNA-associated function may represent the most primal form of innate immunity, allowing individual cells to intracellularly control (at a post-transcriptional but pre-translational level) the expression of 'undesirable' mRNAs of either exogenous or endogenous origins.

Here we will review some of the recent work concerning RBGS and present a novel hypothesis that includes possible alternate mechanisms which may function in vivo to mediate immunity associated with the control of retroelements and transposons (Bagasra 1999, Ketting et al. 1999, Tabara et al. 1999). We shall also offer some thoughts on the significance of these mechanisms from an evolutionary perspective. Indeed, RBGS and related processes may help to clarify some of the genetic processes of evolution, particularly in regard to the 'punctuated' evolution (a mechanism by which a new species appears suddenly) so often observed among eukaryotes of the paleontologic past. These same evolutionary processes may explain why the genomes of virtually all eukaryotes are packed with so-called "junk DNA" (non-coding genes) and why they are being maintained as essential parts of non-coding gene sequences.

\section{The gene silencing concept}

Among eukaryotes, RBGS mechanisms may be the primary molecular defense against retroviruses, pararetroviruses, transposons, retrotransposons, and dsRNA viruses. These agents are fundamentally different from all other infectious particles; retroelements are able to manipulate genomic DNA and insert themselves into a host's genome, occasionally even into the germ line DNA. Over time, retroviral DNA can spread throughout the genome of an entire species. Humans and the majority of eukaryotes carry within their genomes a large repertoire of endogenous retroviruses from their evolutionary past (reviewed in Seitz et al. 2003). Some of these endogenous retroviruses remain surprisingly active, particularly during embryogenesis and oncogenesis (Fire 1999, Sharp 1999; Bass 2000, Seitz et al. 2003).

Besides RNAi, RBGS involves molecular immunity (Bagasra \& Amjad 1997, Bagasra 1999, Bagasra \& Amjad 2000), microRNA (miRNA) (Grishok \& Mello 2002), tncRNA (Ambros 2001), homology-dependent gene silencing (Salo \& Baguna 2002), quelling (Hamilton \& Baulcombe 1999), co-suppression (in Fire 1999, Hamilton \& Baulcombe 1999, Sharp 1999, Tuschl 1999, Bass 2000, Zamore et al. 2000, Grishok \& Mello 2002, Hannon 2002, Hutvagner \& Zamore 2002, Lindenbach \& Rice 2002), RNA dependent DNA methylation (Fire 1999, Hamilton \& Baulcombe 1999, Sharp 1999, Tuschl 1999, Bass 2000, Zamore et al. 2000, Grishok \& Mello 2002, Hannon 2002, Hutvagner \& Zamore 2002, Lindenbach \& Rice 2002), and apparently all host defenses that rely on host-parasite gene sequence homology (Ambros 2001, Ambros et al. 2003). The RBGS mechanism functions to prevent new retroelements, dsRNA viruses, and transposons from entering the host genome. Of note, over $60 \%$ of human gene sequences appear to be retroviral in origin, and over $98 \%$ of the human genome is composed of noncoding RNAs (Bagasra, 1999, Ambros 2001, Ambros et al. 2003). The extended maintenance of retroviral sequences suggests a beneficial function of these sequences through evolution as well as the ability of some of these agents to pass through the molecular defenses, (i.e., the emergence of AIDS for example) (Bagasra 1999, Hamilton \& Baulcombe 1999, Tuschl 1999, Hul et al. 2000). Therefore, this massive accumulation of genomic retroelements may account for its defensive function against newly-arriving retroviruses. By utilizing gene sequences accommodated in the past, the host may preserve its genome from further encroachment by potentially hostile new retroviruses via RBGS. However, these systems appear to exhibit 
flexibility. If a host encounters a retroelement in which the precise homologous siRNAs are not expressed by the endogenous host genome, then the host can accommodate new and evolving retroelements and viruses, thus arming itself with new gene sequences and protecting against subsequent invasion using these new siRNAs. New viruses, transposons, and retroelements periodically emerge via recombination between two or more kinds of retroviruses, transmitted by zoonosis (infection of one species by a microorganism from different species) (reviewed in Bagasra 1999, Ambros 2001, Ambros et al. 2003, Seitz et al. 2003). These siRNAs can be utilized to defend against genetically homologous viruses, and at times the remainder of the uninfected host cell as well (in Bagasra 1999, Fire 1999, Hamilton \& Baulcombe 1999, Sharp 1999, Tuschl 1999, Bass 2000, Zamore et al. 2000, Ambros 2001, Grishok \& Mello 2002, Hannon 2002, Hutvagner \& Zamore 2002, Lindenbach \& Rice 2002, Ambros et al. 2003). Periodically, the acquired gene sequences can be passed on, arming the next generation with these protective genes sequences (Jakowitsch et al. 1999, Hul et al. 2000, Jensen et al. 2002).

The actions of endogenous retroviruses may allow for the reshuffling of genetic sequences within genomes. McClintock's (1950) "jumping genes" are excellent examples of this. It is becoming clear that small hairpin RNAs or microRNAs (miRNAs) participate in the regulation of endogenous retroviruses, as the activity is generally confined to the nucleus. It is interesting how a major portion of non-coding genes, significant numbers of which contain active promoters and fully coding retroelements and mobile genes, are regulated by a finely tuned RBGS system. Control of these reshuffling processes is crucial; while a certain amount of genetic change is desirable, too much is deleterious (Fire 1999, Hamilton \& Baulcombe 1999, Sharp 1999, Tuschl 1999, Bass 2000, Zamore et al. 2000, Grishok \& Mello 2002, Hannon 2002, Hutvagner \& Zamore 2002, Lindenbach \& Rice 2002). Nonetheless, the reshuffling of genetic sequences in large chunks is a critical mechanism in eukaryotic evolution as large changes occur very quickly during adaptation to changing environments. Such a mechanism helps to explain the evolution of novel genetic sequences for which no intermediate forms are evident (Adams et al. 2002).

\section{Transposons and retroelements in the human genome}

The human genome is comparatively more populated with transposable elements than those of other species whose genomes have been analyzed. These "jumping genes" can cause disease and alter the genome in a number of different ways (reviewed in Ostertag \& Kazazian 2001). There are two main classes of transposable elements: DNA transposons and retrotransposons. Retrotransposons are copied into RNA, which is then reverse-transcribed into DNA and inserted into the genome at a new location. These elements therefore expand in number by a duplicative "copy-and-paste" mechanism. The human genome is littered with remnants of the most prominent nonlong-terminal repeat (non-LTR) retrotransposons and human long repetitive elements (LINES), roughly a half million of which are $5^{\prime}$ truncated, inverted, or mutagenically inactivated. However, approximately 5000 are full-length elements of at least $6 \mathrm{~kb}, 60-100$ of which are still capable of retrotransposition. Fulllength elements contain a $5^{\prime}$ untranslated region with an internal promoter, $1 \mathrm{~kb}$ open reading frame (ORF), one ORF1 that encodes a protein with an RNA binding capability, a $4 \mathrm{~kb}$ ORF2 that encodes a protein with endonuclease and reverse transcriptase activities, a short $3^{\prime}$ untransted region, and a poly (A) tail. It is hypothesized that their insertion into chromosomal DNA occurs by the process of target-primed reverse transcription (TPRT), whereby the endonuclease of ORF2 nicks a single strand of DNA and leaves a 3'$\mathrm{OH}$ that serves as the primer for reverse transcription with the L1 RNA as a template. In summary, L1s account directly or indirectly for about one-third of the human genome (Ostertag \& Kazazian 2001).

It has been shown that apparently endogenous retroviruses can "pop out" of a genome and again become infectious particles carrying active packets of genetic information. These particles bear the potential to cross species barriers, as ongoing studies of the historically recent HIV-1 epidemic clearly demonstrate (reviewed in Bagasra 1999).

\section{Amplification, communication, and epigenetic persistence of RNAi}

It is clear that either endogenous or exogenous small ( 22 bp) dsRNAs initiate a cascade of events that allow enzymes such as Dicer (or RNaseIII or its equivalent, Bernstein et al. 2003) to cleave the target mRNA into 22 bp fragments (Hul et al. 2000, Ambros 2001, Seitz et al. 2003). The annealing reaction between double stranded siRNAs and single stranded target mRNA forms a triplex complex. RNA-dependent RNA polymerase amplifies these "silencing complexes" that are cast off in the helicase reaction. This step involves the formation of the ATP-helicase-triplex complex that culminates in the unwinding and release of complex siRNAs (Fire 1999, Hamilton \& Baulcombe 1999, Sharp 1999, Tuschl 1999, Bass 2000, Zamore et al. 2000, Grishok \& Mello 2002, Hannon 2002, Hutvagner \& Zamore 2002, Lindenbach \& Rice 2002). These siRNAs bind mRNA and generate more siRNAs while degrading target mRNA. The resulting 


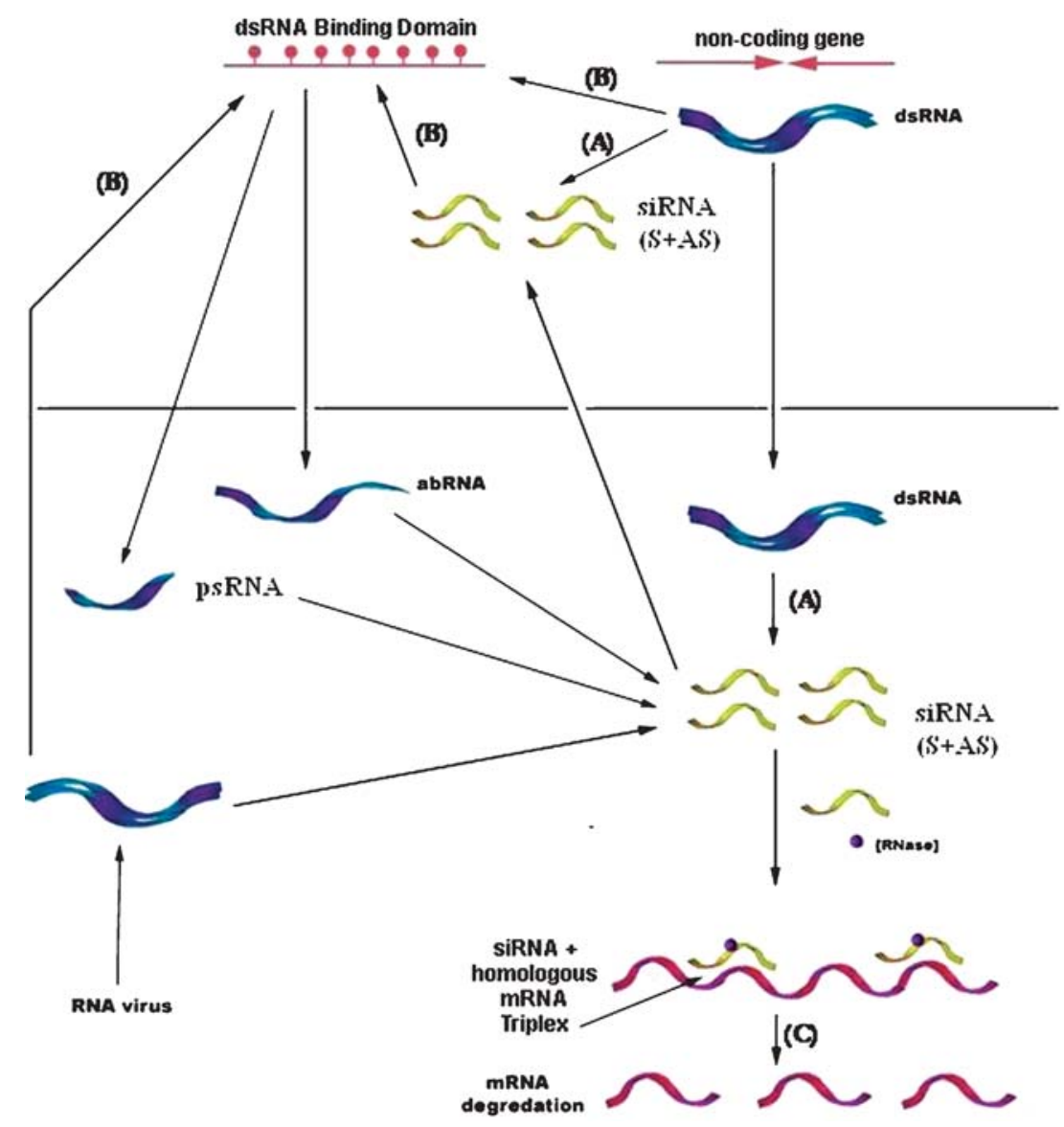

Figure 1. A model for the mechanism of mRNA degradation by RNAi. Small interfering RNAs (siRNAs) are the result of non-coding RNAs, part of introns, and other endogenous retroelements, accumulated throughout evolution. These pre-siRNAs are expressed as dsRNA a part of the large transcripts (blue ribbons) and broken down to small fragments of $\sim 22$ bp as siRNAs (yellow ribbons: step A). These siRNAs remain as dsRNA: sense (S) and antisense (AS). In the initial step (A), the dsRNA that initiates RNAi is bound by the RNAi nuclease (not shown), and then degraded to small dsRNAs (siRNAs) that remain stably bound to the RNAi nuclease. The enzymes coat the dsRNA in a precise register to generate specific fragments directing the cleavage of the mRNA at specific sites. When a retrovirus or other genetic element enters the cells, the siRNAs bind to the homologous gene sequences of the invading virus. This homologous binding forms a triplex and initiates a cascade of events resulting in the generation and amplification of siRNAs. The reaction is catalyzed by several enzymes including a Dicer (or RNA-dependent RNA Polymerase III or equivalent) that contains a dsRNA binding domain (red knob) on one or more ribonuclease domains (B), as well as an RNA helicase domain. In the subsequent step (C), these small template sequence-specific pieces cleave the mRNA. The helicase domain of the protein catalyzes an ATP-dependent strand exchange, replacing the sense stand(s) of the small dsRNA template with the mRNA. The mRNA is then cleaved to regenerate the RNAi nuclease with its small dsRNA. These siRNAs recycle and are amplified, transmitted to other cells, and silence the gene of interest in those cells. In addition, these RNA-based gene-silencing mechanisms also degrade aberrant RNA transcripts (abRNA) as well as pseudogenes (psRNA), and RNA viruses.

fragments, which are specific to the gene being silenced, may be distributed widely throughout the organism. The experimental data clearly show that the RBGS function is accompanied by the development and accumulation of significant quantities of siRNA fragments. Such has been demonstrated via Northern blotting techniques (Fire 1999, Hamilton \& Baulcombe 1999, Sharp 1999, Tuschl 1999, Bass 2000, Zamore et al. 2000, Grishok \& Mello 2002, Hannon 2002, Hutvagner \& Zamore 2002, Lindenbach \& Rice 2002). Furthermore, in both C. elegans and Neurospora, the memory of specific RNA sequences that have been silenced persists epigenetically through several generations. The signal then seems to fade if the progeny are not "challenged" by the mRNA in question (Fire 1999, Hamilton \& Baulcombe 1999, Sharp 1999, Tuschl 1999, Bass 2000, Zamore et al. 2000, Grishok \& Mello 2002, Hannon 2002, Hutvagner \& Zamore 2002, Lindenbach \& Rice 2002). In these species siRNA signals are readily transmitted from cell to cell, although the mechanisms of heritability and intracellular communication underlying these observations are not yet explained (Figure 1. Fire 1999, Hamilton \& Baulcombe 1999, Ketting et al. 1999, Sharp 1999, Tabara et al. 1999, Tuschl 1999, Bass 2000, Zamore et al. 2000, Grishok \& Mello 2002, Hannon 2002, Hutvagner \& Zamore 2002, Lindenbach \& Rice 2002, Salo et al. 2002, Urwin et al. 2002). 


\section{A unified hypothesis of RBGS mechanisms}

RNAi, miRNA, tncRNA, molecular immunity, homology-dependent gene silencing, and DNA dependent methylation are host defenses that rely on homologous host-retroelement gene sequences (Bagasra 1999, Fire 1999, Hamilton \& Baulcombe 1999, Ketting et al. 1999, Sharp 1999, Tabara et al. 1999, Tuschl 1999, Bass 2000, Zamore et al. 2000, Grishok \& Mello 2002, Hannon 2002, Hutvagner \& Zamore 2002, Lindenbach \& Rice 2002, Salo et al. 2002, Urwin et al. 2002). These defenses have evolved to accommodate both the host and the parasite (Bagasra \& Amjad 1997, Bagasra 1999, Fire 1999, Hamilton \& Baulcombe 1999, Ketting et al. 1999, Sharp 1999, Tabara et al. 1999, Tuschl 1999, Bagasra \& Amjad 2000, Bass 2000, Zamore et al. 2000, Grishok \& Mello 2002, Hannon 2002, Hutvagner \& Zamore 2002, Lindenbach \& Rice 2002, Salo et al. 2002, Urwin et al. 2002). The RBGS mechanism functions to prevent new retroelements and transposons from entering the host genome. The extensive retention of retroviral sequences in eukaryotic life forms suggests a beneficial function for these sequences in the course of evolution (Ambros 2001, Ambros et al. 2003, Seitz et al. 2003). Therefore, this massive accumulation of retroelements in eukaryote genomes may account for its defensive function against newlyarriving retroviruses. We have hypothesized that noncoding sequences are expressed in the form of siRNAs (Bagasra et al. 1993, Bagasra \& Amjad 1997, Bagasra 1999, Bagasra \& Amjad 2000). Recently, Ambros and colleaguese (Carrington \& Ambros, 2003) have analyzed miRNAs and tncRNAs in $C$. elegans and have discovered that among the short cDNA sequences that correspond to protein-coding sequences, only 49 were from the sense strand of an mRNA, whereas 746 distinct cDNA sequences were antisense to the coding strand of mRNAs. They reported that the siRNAs that accumulate in $C$. elegans in response to exogenous dsRNA triggers are also primarily antisense to the targeted mRNA (Ambros 2001, Ambros et al. 2003, Carrington \& Ambros 2003), suggesting that the antisense sequences they identified represent endogenous siRNAs. A similar result was obtained for the plant Arabidopsis thaliana, where a significant fraction of $\sim 22 \mathrm{nt}$ cDNAs that were analyzed corresponded to protein coding sequences (reviewed in Carrington \& Ambros 2003). In the Arabidopsis case, cDNA sequences were obtained for either the sense or antisense orientation relative to the open reading frame, which is consistent with the duplex nature of plant siRNAs (Carrington \& Ambros 2003).

It appears that in using gene sequences accommodated in the past, the host protects its genome against encroachment by potentially hostile new retroviruses via the RBGS system. However, these systems appear to be flexible. If a host encounters a parasite in which the precise homologous siRNAs are not expressed by the host genome, then the host has the ability to accommodate new and evolving retroelements and viruses, arming the host with new gene sequences and thus protecting against the next invasion via these new siRNAs. New viruses, transposons, and retroelements are known to emerge periodically by recombination between two or more kinds of retroviruses and by zoonosis (reviewed in Gitlin \& Andino 2003). These non-coding siRNAs can be utilized to defend against invasions by viruses that carry homologous gene sequences, and at times the remainder of the uninfected host cell as well (Bagasra 1999, Bagasra et al. 1993, Carrington et al. 2003, Seitz et al. 2003). In some cases, the newly acquired gene sequences can be passed on to future generations (Carrington \& Ambros, 2003).

\section{Role of siRNAs in development of HIV-1 vaccine}

The ultimate and most dramatic use of RBGS would be to silence HIV-1 genes. Why are naturally occuring siRNAs not effective against HIV-1, yet are likely able to protect chimpanzees against SIVcpz? The solution most likely derives from understanding how chimpanzees, which are genetically close to humans, are able to remain healthy after SIVcpz (which bears $>90 \%$ homology to HIV-1) infection, whereas humans are susceptible to HIV-1. There are a few possible explanations to this puzzle. For example, chimps in the wild carry SIVcpz and show a low to moderate viral load yet appear to be immune to this lentivirus against the development of AIDS (Ondoa et al. 2001). Similarly, several African non-human primates carry various versions of SIVs but remain unaffected by them. How do they become resistant to SIVs? One possibility may be that all the African primates acquired lentiviruses by slow exposure to various pre-lentiviruses over millions of years. They could have accumulated and amplified protective siRNAs without significant population impact. The accumulation of new siRNAs most likely took place when they invaded other primate colonies. These newly acquired viral sequences could then incorporated into their somatic cells as well as being partially or completely silenced (depending upon the repertoire of siRNAs that their respective genomes were expressing at the time of exposure); after many generations these new sequences were then incorporated into their germ lines, endowing their progeny with resistance to newer lentiviral sequences. That chimps and other African primates incompletely block their natural SIVs suggests that these viruses may be serving an evolutionary protective role by replicating at low levels to keep the RBGS mechanism activated until homologous genes are incorporated into the germ line. 
The chief reason to entertain the notion that SIV gene fragments accumulated in the African primates slowly and did not result in high mortality is the observation that, in captivity, many African primates are exposed to different kinds of lentiviruses but without any major illness (reviewed in Bagasra 1999, Muchmore 2001). Humans also follow a similar course of events upon exposure to a single strain of SIV. For example, there are cases of accidental monkey bites in Africa and elsewhere in the world, primarily among animal care workers. However, they have not led to an HIV-1 like illness or epidemic (Bagasra 1999). Similarly, many African tribes rely upon primates, including chimpanzees, as their main diet. There is no evidence, however, that these tribes carry SIVs or have even seroconverted to a significant degree during the process, suggesting that natural exposure to a small dose of a single SIV strain in human or non-human primates (through bites) causes no significant illness.

HIV-1 may have arisen from massive recombination events between various SIVs (SIVcpz, SIVsm and SIVagm: reviewed in Salemi et al. 2003, Apetrei et al. 2004) that resulted in numerous new forms of lentiviral sequences via zoonosis (Salemi et al. 2003). Several of these new lentiviruses became infectious because the human host lacked matching endogenous siRNAs with which to neutralize them (Bagasra 1999). We hypo thesize that, after the accidental introduction into human populations, these new lentiviral sequences went through a selection process, and those least homologous to the human RBGS (siRNAs) gave rise to the most successful versions of HIV-1 (this is similar to recombinant BSV described by Jakowitsch et al. 1999, Hul et al. 2000, Jensen et al. 2002). Since there is a great deal of variation between human populations with regard to endogenous siRNAs, they may be expressing different sequences of siRNAs and therefore the success rate of various HIV-1 clades differ significantly from one geographic locale to another (reviewed in Otting et al. 2002). This selection is constantly taking place, and new recombinants are constantly adapting to evade the pre-existing molecular defenses (Robertson et al. 1995, Sharp et al. 2000). Since germline integration into the human genome is rare, the natural integration of the new protective sequences will most likely occur very slowly.

Interestingly, one would expect that a few groups or individuals who are exposed to low doses of multiple strains of HIV-1 (or SIVcpz) would have a better opportunity to activate and amplify specific siRNAs against HIV-1 quasispecies, and hence would be more resistant to HIV-1 than an individual who was exposed to a single strain of a large dose of HIV-1. In recent years, several reports have described HIV-1 resistant individuals among African sex workers (Kaul et al. 2001, Messele et al. 2001). These individuals are regularly exposed to various HIV-1 strains due to unprotected sex (reviewed by Kaul et al. 2002). Numerous additional reports indicate that certain individuals or groups are immune to HIV-1 altogether (Baur et al. 1989, Bryson et al. 1995, Deacon et al. 1995, Roques et al. 1995, Paxton et al. 1996, Kaul et al. 2000, Kulkarni et al. 2003), while others remain AIDS free for extensive periods of time (Otting et al. 2002). In order to develop sufficient human protection against HIV-1 variants, a number of homologous sequences will be required in the endogenous repertoire (Hiroshika et al. 2000, Donze \& Picard 2002, Hohjoh 2002, Jeong et al. 2002, Leirdal \& Sioud 2002, Miyagishi \& Taira 2002, Paddison et al. 2002, Paul et al. 2002, Sui et al. 2002, Yu et al. 2002). Through genetic technology, however, a rapid HIV-1 inhibitory siRNAs specific repertoire can perhaps be designed. These siRNAs could be encoded and delivered by appropriate vectors and then utilized in preventative or therapeutic vaccines (Hiroshika et al. 2000, Donze \& Picard 2002, Hohjoh 2002, Jeong et al. 2002, Leirdal \& Sioud 2002, Miyagishi \& Taira 2002, Paddison et al. 2002, Paul et al. 2002, Sui et al. 2002, Yu et al. 2002). In conclusion, we are optimistic that the RBGS system could be utilized therapeutically to silence HIV-1.

\section{Issues with current models of RNAi}

Virtually all of the data published to date have been generated using experimental models in which the RBGS mechanism is examined with artificial dsRNA strands that are generated either in vitro or by vectors producing dsRNA upon transfection/transduction (reviewed in Hamilton \& Baulcombe 1999, Fire 1999, Sharp 1999, Tuschl 1999, Bass 2000, Zamore et al. 2000, Grishok \& Mello 2002, Hannon 2002, Hutvagner \& Zamore 2002, Lindenbach \& Rice 2002). Meaningful translation of in vitro data must, however, be approached with caution: to date, there has been no clear description of an in vivo model analogous to the siRNA effect. This is somewhat unusual, given the considerable years of experimentation as well as the extraordinary ubiquity of the observed mechanism in a wide variety of life forms (Hamilton \& Baulcombe 1999, Fire 1999, Ketting et al. 1999, Sharp 1999, Tabara et al. 1999, Tuschl 1999, Bass 2000, Zamore et al. 2000, Grishok \& Mello 2002, Hannon 2002, Hutvagner \& Zamore 2002, Lindenbach \& Rice 2002, Salo et al. 2002, Urwin et al. 2002). Therefore, the extent of the role played by RBGS as an antiviral system remains to be fully characterized. It is unclear whether RNA silencing can be elicited naturally after exposure to retroelements, transposons or dsRNA viruses. Some of the critical questions that must be addressed include:

(i) Are siRNAs generated during natural infection with retroelements and transposons? Reactivation of 
transposons after the siRNA generating system supports this notion (Ketting et al. 1999, Tabara et al. 1999), but more work is needed before this issue is resolved.

(ii) Are RBGS components upregulated during viral infection? Several critical enzymes have been identified which participate in the RBGS system. These include Dicer, RNA-dependent RNA polymerase (RdRP), RNA degradation enzymes, and several other enzymes (reviewed in Grishok \& Mello 2002). The upregulation of siRNA-generating components during viral infection would support the role of this system in antiviral defense.

(iii) Have viruses evolved mechanisms to suppress or escape a RBGS system? Several viral proteins have been identified which can quench the RBGS system (reviewed in Gitlin \& Andino 2003), but more evidence is needed.

(iv) Can RBGS in one infected cell trigger a systemic antiviral response? In $C$. elegans, injection of dsRNA into one region of the worm triggers its spread to many different tissues, including the gonad (Urwin et al. 2002). Similar observations were made for plants (Hamilton \& Baulcombe 1999, Salo \& Baguna 2002) and mammalian cells, including human (Donze \& Picard 2002, Hohjoh 2002, Leirdal \& Sioud 2002, Miyagishi \& Taira 2002, $\mathrm{Yu}$ et al. 2002). It is not known how the dsRNAs exit the cell in which they are produced, how they are systemically disseminated, or how they are taken up by distant target cells. It will thus be important to address whether RNA silencing in mammals also induces a systemic response. Few reports have yet suggested this to be the case (Desset et al. 2003); additional studies are needed.

(v) What is the origin of RBGS? One way to answer this question is to evaluate what happens to an organism when certain genes, presumed to be involved in RBGS, are altered. Mutations in several genes render an organism incapable of generating siRNAs; in some cases, these mutations reactivate transposons and retroelements (Ketting et al. 1999, Tabara et al. 1999, Hirochika et al. 2000, Nakayashiki et al. 2001, Jeong et al. 2002, Paddison et al. 2002, Paul et al. 2002, Sui et al. 2002). Proteins essential for the homologous RNA degradation pathway are highly conserved in fungi, plants, vertebrates, and mammals (reviewed in Grishok \& Mello 2002). These proteins have been shown to play essential roles in organismal development, germline fate, and host defenses against both transposable elements and dsRNA viruses. However, the presence of these proteins and genes may be a small piece of a large puzzle. The real siRNA sequences involved in RBGS may be hidden from our eyes. How they are expressed, and how is the expression process coordinated? We will need deeper probing to solve this mystery.

(vi) Does an adoptive immune system, in which genetic recombination occurs among a limited number of genes (i.e., VDJ), similarly lead to diversity within the siRNA generating mechanism? If this is true, then one ought to be able to recognize the homologous enzymes and proteins involved, such as: RAG1/RAG2-recombination enzymes, ligase IV, helicase, and the dsDNA restriction and splicing elements (reviewed in Martin 2002).

(vii) In what form are siRNAs transported? Are $\operatorname{ss} R A$, $d S R N A$ or triplex siRNA or nucleic acid-complexs transported from cell-to-cell? Our current understanding of molecular biology would suggest that the safest and most stable mode of transmission of molecular immunity would be in the form of triplex complexes (Bagasra 1999), since ss and ds nucleic acids can be readily digested by numerous nucleases active intracellularly or extracellularly. However, currently there is no nuclease known that digests triplex nucleic acid complex. This important issue still remains essentially uninvestigated.

We are unaware of conclusive evidence thus far of persistent double-stranded RNAs that exist naturally, other than in certain transient or rare circumstances involving viral activity, or in an infection with a relatively rare class of viruses that utilize dsRNA (such as Reoviridae, Orbivirus, Cyoviruses-or the once obscure West Nile virus). Furthermore, in these viral examples, the dsRNA is always encapsulated in a viral protein structure while in the host cell cytoplasm. The dsRNA is therefore not readily available for interaction with the normal enzymes or siRNA of the cell (Gitlin \& Andino, 2003). For example, the bluetongue virus produces capped mRNA internally within an enclosed core particle, releasing only ssRNA into the cytoplasm of the host cell. Even more intriguing is that some of the members of the Orthoreovirus carry a protein designed to quench any dsRNA that might have been released accidentally into the cellular cytoplasm, thereby avoiding detection by an RNAi defense system (Mallory et al. 2001, Gitlin \& Andino 2003).

Nonetheless, double-stranded RNA is always required in the various experiments cited in order to stimulate the RBGS response. All of the RNAi data reviewed thus far should therefore be viewed in light of this in vitro component, in which a type of "molecular mimicry" may actually be occurring. Instead, we hypothesize that these experimental protocols are actually simulating a natural system of a slightly different function that evolved for specific purposes. It appears that in eukaryotes, this system is primarily a molecular 
immune mechanism specializing in fighting retroviruses, pararetroviruses, transposons, retrotransposons, aberrant transcripts, and protecting unnecessary gene arrangements (Hamilton \& Baulcombe 1999, Fire 1999, Ketting et al. 1999, Sharp 1999, Tabara et al. 1999, Tuschl 1999, Bass 2000, Zamore et al. 2000, Grishok \& Mello 2002, Hannon 2002, Hutvagner \& Zamore 2002, Lindenbach \& Rice 2002, Salo et al. 2002, Urwin et al. 2002, Seitz et al. 2003).

\section{References}

Adams KL, Qiu YL, Stoutemyer M, Palmer JD (2002) Punctuated evolution of mitochondrial gene content: high and variable rates of mitochondrial gene loss and transfer to the nucleus during angiosperm evolution. Proc Natl Acad Sci 99: 9905-9912.

Apetrei C, Robertson DL, Marx PA (2004) The history of SIVS and AIDS: epidemiology, phylogeny and biology of isolates from naturally SIV infected non-human primates (NHP) in Africa. Front Biosci 9: 225-254.

Ambros V (2001) MicroRNAs: tiny regulators with great potential. Cell 107: 823-826.

Ambros V, Lee RC, Lavanway A, Williams PT, Jewell D (2003) Micro RNAs and Other Tiny Endogenous RNAs in C. elegans. Curr Biol 13: 807-818.

Bagasra O (1999) In HIV and Molecular Immunity: Prospect for AIDS Vaccine. Eaton publishing, Natick, MA pp. 22-28.

Bagasra O, Amjad M (1997) Natural immunity against HIV-1: Prospect for AIDS vaccine. Front Biosci 2: 387-402.

Bagasra O, Amjad M (2000) Protection against Retroviruses are owing to a different form of immunity: A RNA- Based Molecular Immunity hypothesis. Appl Immunochem Mol Morphol 8: 133-146.

Bagasra O, Pomerantz RJ, Whittle P (1993) The role of CD8 + lymphocytes on unstimulated peripheral blood lymphocytes to infection with HIV-1. Immunol Letters 35: 83-92.

Bass BL (2000) Double-stranded RNA as a template for gene silencing. Cell 101: 235-238.

Baur A, Schwarz N, Ellinger S, Korn K, Harrer T, Mang K, Jahn G (1989) Continuous clearance of HIV in a vertically infected child. Lan 2: 1045.

Beattie T, Rowland-Jones S, Kaul R (2002) HIV-1 and AIDS: what are protective immune responses? J HIV Ther 7: 35-39.

Bernstein E, Kim SY, Carmell MA, Murchison EP, Alcorn H, Li MZ, Mills AA, Elledge SJ, Anderson KV, Hannon GJ (2003) Dicer is essential for mouse development. Nat Genet. 35: 215-217.

Boden D, Pusch O, Lee F, Tucker L, Ramratnam B (2003) Human immunodeficiency virus type 1 escape from RNA interference. $J$ Virol 77: 11531-11535.

Bryson YJ, Pang S, Wei LS, Dickover R, Diagne A, Chen IS (1995) Clearance of HIV infection in a perinatally infected infant. New Engl $J$ 332: 833-838.

Carrington JC, Ambros V (2003) Role of microRNAs in plant and animal development. Science 301: 336-338.

de Groot NG, Otting N, Doxiadis GG, Balla-Jhagjhoorsingh SS, Heeney JL, van Rood JJ, Gagneux P, Bontrop RE (2002) Evidence for an ancient selective sweep in the MHC class I gene repertoire of chimpanzees. Proc Natl Acad Sci USA 99: 11748-11753.

Deacon NJ, Tsykin A, Solomon A, Smith K, Ludford-Menting M, Hooker DJ, McPhee DA, Greenway AL, Ellett A, Chatfield C (1995) Genomic structure of an attenuated quasispecies of HIV-1 from a blood transfusion donor and recipients. Sci 270: 988-991.

Desset S, Meignin C, Dastugue B, Vaury C (2003) COM a Heterochromatic Locus Governing the Control of Independent Endoge- nous Retroviruses From Drosophila melanogaster . Genet 164: $501-509$.

Donze O, Picard D (2002) RNA interference in mammalian cells using siRNAs synthesized with T7 RNA polymerase. Nucleic Acids Res 30: e46.

Fire A (1999) RNA-triggered gene silencing. Trends Genet 15: 358363.

Gitlin L, Andino R (2003) Nucleic acid-based immune system: the antiviral potential of mammalian RNA silencing. $J$ Virol 77: 71597165.

Grishok A, Mello CC (2002) RNAi (Nematodes: Caenorhabditis elegans). Adv Genet 46: 339-360.

Hamilton AJ, Baulcombe DC (1999) A species of small antisense RNA in posttranscriptional gene silencing in plants. Sci. 286: 950952.

Hannon GJ (2002) RNA interference. Nat 418: 244-251.

Hirochika H, Okamoto H, Kakutani T (2000) Silencing of retrotransposons in Arabidopsis and reactivation by the ddm1 mutation. Plant Cell 12: 357-369.

Hohjoh H (2002) RNA interference (RNAi) induction with various types of synthetic oligonucleotide duplexes in cultured human cells. FEBS Lett 521: 195-199.

Hu WY, Myers CP, Kilzer JM, Pfaff SL, Bushman FD (2002) Inhibition of retroviral pathogenesis by RNA interference. Curr Biol 12: $1301-1311$.

Hull R, Harper G, Lockhart B (2000) Viral sequences integrated into plant genomes. Trends Plant Sci 5: 362-265.

Hutvagner G, Zamore PD (2002) RNAi: nature abhors a doublestrand. Curr Opin Genet Dev 12: 225-232.

Jacque JM, Triques K, Stevenson M (2002) Modulation of HIV-1 replication by RNA interference. Nat 418: 435-438.

Jakowitsch J, Mette MF, van Der Winden J, Matzke MA, Matzke AJ (1999) Integrated pararetroviral sequences define a unique class of dispersed repetitive DNA in plants. Proc Natl Acad Sci 96: 13241-13246.

Jensen S, Gassama MP, Dramard X, Heidmann T (2002) Regulation of $I$-transposon activity in Drosophila: evidence for cosuppression of nonhomologous transgenes and possible role of ancestral $I$ related pericentromeric elements. Genet 162: 1197-1209.

Jeong Br BR, Wu-Scharf D, Zhang C, Cerutti H (2002) Suppressors of transcriptional transgenic silencing in Chlamydomonas are sensitive to DNA-damaging agents and reactivate transposable elements. Proc Natl Acad Sci 99: 1076-1081.

Joost Haasnoot PC, Cupac D, Berkhout B (2003) Inhibition of virus replication by RNA interference. J Biomed Sci 10: 607-616.

Kaul R, Plummer FA, Kimani J, Dong T, Kiama P, Rostron T, Njagi E, MacDonald KS, Bwayo JJ, McMichael AJ, RowlandJones SL (2000) HIV-1-Specific Mucosal CD8 + Lymphocyte Responses in the Cervix of HIV-1-Resistant Prostitutes in Nairobi. J Immunol 164: 1602-1611.

Ketting RF, Haverkamp TH, van Luenen HG, Plasterk RH (1999) Mut-7 of C. elegans, required for transposon silencing and RNA interference, is a homolog of Werner syndrome helicase and RNaseD. Cell 99: 133-141.

Kulkarni PS, Butera ST, Duerr AC (2003) Resistance to HIV-1 infection: lessons learned from studies of highly exposed persistently seronegative (HEPS) individuals. AIDS Rev 5: 87-103.

Leirdal M, Sioud M (2002) Gene silencing in mammalian cells by preformed small RNA duplexes. Biochem Biophys Res Common 295: 744-748.

Lindenbach BD, Rice CM (2002) RNAi targeting an animal virus: news from the front. Mol Cell 9: 925-927.

Mallory AC, Ely L, Smith TH, Marathe R, Anandalakshmi R, Fagard M, Vaucheret H, Pruss G, Bowman L, Vance VB (2001) HC-Pro suppression of transgene silencing eliminates the small RNAs but not transgene methylation or the mobile signal. Plant Cell 13: 571-583. 
Marin L, Lehmann M, Nouaud D, Izaabel H, Anxolabehere D, Ronsseray S (2000) P-Element repression in Drosophila melanogaster by a naturally occurring defective telomeric P copy. Genetics 155: $1841-1854$

Martin G (2002) V(D)J Recombination: RAG proteins, repair factors, and regulation. Annu Rev Biochem 71: 101-132.

McClintock B (1950) The origin and behavious of mutable loci in maize. Proc Natl Acad Sci 36: 347.

Messele T, Rinke de Wit TF, Brouwer M, Aklilu M, Birru T, Fontanet AL, Schuitemaker H, Hamann D (2001) No difference in in vitro susceptibility to HIV type 1 between high-risk HIV-negative Ethiopian commercial sex workers and low-risk control subjects. AIDS Res Hum Retroviruses.17: 433-441.

Miyagishi M, Taira K (2002) U6 promoter driven siRNAs with four uridine $3^{\prime}$ overhangs efficiently suppress targeted gene expression in mammalian cells. Nat Biotechnol 20: 497-500.

Muchmore EA (2001) Chimpanzee models for human disease and immunobiology. Immunol Rev 183: 86-93.

Nakayashiki H, Ikeda K, Hashimoto Y, Tosa Y, Mayama S (2001) Methylation is not the main force repressing the retrotransposon MAGGY in Magnaporthe grisea. Nucleic Acids Res 29: $1278-1284$.

Ondoa P, Kestens L, Davis D, Vereecken C, Willems B, Fransen K, Vingerhoets J, Zissis G, ten Haaft P, Heeney J, van der Groen G (2001) Longitudinal comparison of virus load parameters and CD8 T-cell suppressive capacity in two SIVcpz-infected chimpanzees. J Med Primatol 30: 243-253.

Ostertag EM, Kazazian HH Jr (2001) Biology of mammalian L1 retrotransposons. Annu Rev Genet 35: 501-538.

Otting N, de Groot NG, Doxiadis GG, Bontrop RE (2002) Extensive Mhc-DQB variation in humans and non-human primate species. Immunogenetics 54: 230-239.

Paddison PJ, Caudy AA, Bernstein E, Hannon GJ, Conklin DS (2002) Short hairpin RNAs (shRNAs) induce sequence-specific silencing in mammalian cells. Genes Dev 16: 948-958.

Paul CP, Good PD, Winer I, Engelke DR (2002) Effective expression of small interfering RNA in human cells. Nat Biotechnol 20: 505-508.

Paxton WA, Martin SR, Tse D, O'Brien TR, Skurnick J, VanDevanter NL, Padian N, Braun JF, Kotler DP, Wolinsky SM, Koup RA (1996) Relative resistance to HIV-1 infection of CD4 lymphocytes from person who remain uninfected despite multiple highrisk sexual exposure. Nat Med 2: 412-417.
Robertson DL, Sharp PM, McCutchan FE, Hahn, BH (1995) Recombination in HIV-1. Nat 374: 124-126.

Robin S, Chambeyron S, Bucheton A, Busseau I (2003) Gene silencing triggered by non-LTR retrotransposons in the female germline of Drosophila melanogaster. Genet 164: 521-531.

Roques PA, Gras G, Parnet-Mathieu F, Mabondzo AM, Dollfus C, Narwa R, Marce D, Tranchot-Diallo J, Herve F, Lasfargues G (1995) Clearance of HIV infection in 12 perinatally infected children: clinical, virological and immunological data. AIDS 9: F19-F26.

Salemi M, Oliveira T.De, Courgnaud T, Moulton V, Holland B, Cassol S, Switzer WM, Vandamme AM (2003) Mosaic Genomes of the Six Major Primate Lentivirus Lineages Revealed by Phylogenetic Analyses. J Virol 77: 7202-7213.

Salo E, Baguna J (2002) Regeneration in planarians and other worms: new findings, new tools, and new perspectives. $J$ Exp Zool 292: $528-539$.

Seitz H, Youngson N, Lin SP, Dalbert S, Paulsen M, Bachellerie JP, Ferguson-Smith AC, Cavaille J (2003) Imprinted microRNA genes transcribed antisense to a reciprocally imprinted retrotransposonlike gene. Nat Genet 34: 261-262.

Sharp PM, Bailes E, Robertson DL, Gao F, Hahn BH (2000) Origins and evolution of AIDS viruses. Biol Bull 196: 338-342.

Sharp PA (1999) RNAi and double-strand RNA. Genes Dev 15: 139-141.

Sui G, Soohoo C, Affarel B, Gay F, Shi Y, Forrester WC, Shi Y (2002) A DNA vector-based RNAi technology to suppress gene expression in mammalian cells. Proc Natl Acad Sci 99: 5515-5520.

Tabara H, Sarkissian M, Kelly WG, Fleenor J, Grishok A, Timmons L, Fire A, Mello CC (1999) The rde-1 gene, RNA interference, and transposon silencing in C. elegans. Cell 99: 123-132.

Tuschl T (1999) Targeted mRNA degradation by double-stranded RNA in vitro. Genes Dev 13: 3191-3197.

Urwin PE, Lilley CJ, Atkinson HJ (2002) Ingestion of doublestranded RNA by preparasitic juvenile cyst nematodes leads to RNA interference. Mol Plant Microbe Interact 15: 747-752.

Yu JY, DeRuiter SL, Turner DL (2002) RNA interference by expression of short-interfering RNAs and hairpin RNAs in mammalian cells. Proc Natl Acad Sci 99: 6047-6052.

Zamore PD, Tuschl T, Sharp PA, Bartel DP (2000) RNAi: doublestranded RNA directs the ATP-dependent cleavage of mRNA at 21-23 nucleotide intervals. Cell 101: 25-33. 